\title{
Sur l'idéologie française \\ [Critique de Raymond Aron au livre L'idéologie française de Bernard-Henri Lévy.]
}

\section{Provocation $^{1}$}

Un auteur qui emploie volontiers les adjectifs infâme ou obscène pour qualifier les hommes et les idées invite le critique à lui rendre la pareille. Je résisterai autant que possible à la tentation, bien que le livre de Bernard-Henri Lévy présente quelques-uns des défauts qui m'horripilent : la boursouflure du style, la prétention à trancher des mérites et démérites des vivants et des morts, l'ambition de rappeler à un peuple amnésique la part engloutie de son passé, les citations détachées de leur contexte et interprétées arbitrairement. Pis encore, le doute subsiste à la fin de la lecture : la violence du ton, maintenue d'un bout à l'autre du pamphlet, révèle-t-elle une indignation authentique ou le goût du scandale et de la diffusion de masse?

Allons plus loin : le livre ne se prête guère à une discussion objective, selon le mot consacré dans les universités. II n'apporte aucun fait, aucun document, aucun texte que l'on ne trouve dans les quelques livres dont Bernard-Henri Lévy a tiré, pour l'essentiel, la matière qu'il triture à sa manière. Ce qui lui appartient en propre, c'est une certaine mise en place d'un corpus de mots ou de phrases. Or, cette mise en place est à tel point commandée par le propos de l'auteur que l'on se demande s'il vaut la peine de discuter avec un « philosophe » qui s'arroge le rôle de justicier.

En quoi consiste le propos? D'abord, un procès du pétainisme et de la Révolution nationale, en particulier la mise en cause d'Espritet d'Uriage. A partir de là, la définition de l'idéologie française - non pas « une », mais «la » - dont Vichy révéla la persistance et la force souterraine, d'autant plus menaçante aujourd'hui que le fascisme français ne fut pas épuré à la Libération.

Le vichysme ou le pétainisme, nous le savons, et je le sais depuis 1940, fait partie intégrante de l'histoire politique de la France. Certes, sans la défaite et l'occupation de la moitié du pays par les Allemands, probablement les artisans de la Révolution nationale n'auraient-t-ils jamais accédé au pouvoir. L'Action française exerçait une grande influence sur les officiers de la Marine nationale (ou Royale), dans des milieux limités, mais qui souvent occupaient des positions clefs (sans compter l'Académie); en revanche, elle ne parvenait pas à faire élire un seul député sous la IIIe République (une seule fois, Léon Daudet siégea à la chambre). Or, pendant les premiers mois après la défaite, c'est la pensée de l'Action française qui, par l'intermédiaire de Raphaël Alibert, René Gillouin, et d'autres encore, domina Vichy et inspira les lois effectivement " infâmes » (le statut des Juifs, la remise en question des naturalisations postérieures à 1927, la suppression de la loi qui avait été votée en 1938 pour lutter contre la propagande hitlérienne et l'exploitation des haines raciales).

La conception même d'une Révolution nationale sous le regard des vainqueurs, alors que la guerre continuait, me parut, à l'époque même, non pas monstrueuse ou obscène, mais déraisonnable. En effet, ou bien le IIIe Reich l'emporterait, et, en ce cas, les vichystes seraient balayés par les vrais nazis ; ou bien le IIIe Reich

1 Publiée dans le magazine L'Express le 07/02/1981 [Nota del editor]. 
serait finalement vaincu, et, en ce cas aussi, les vichystes disparaîtraient au profit des ci-devant de la IIIe et d'une nouvelle élite.

Le phénomène Révolution nationale n'eut d'équivalent dans aucun pays de l'Europe occupée, de même que nulle part ailleurs un Etat légal ne s'interposa entre les autorités d'occupation et la population. Les maîtres de cet Etat ne se contentèrent pas d'administrer, ils voulurent régénérer la nation, renouveler les institutions, préparer l'après-guerre, que la plupart des vichystes, dans l'année 1940, croyaient tout proche. La masse de la population, traumatisée par une défaite à l'avance inconcevable, s'accrocha au Maréchal et souscrivit à la condamnation du "régime aboli ». Or, pendant les années 30, nombre de groupes et d'intellectuels, Esprit et Ordre nouveau, Emmanuel Mounier, Arnaud Dandieu et Robert Aron, menaient campagne contre les démocraties capitalistes, alors que montaient, à travers tout l'Europe, les régimes autoritaires ou totalitaires.

«Esprit » s'efforça de prendre ses distances par rapport au fascisme et au national-socialisme, non sans peine, parce qu'il partageait avec eux les mêmes ennemis.

Après la défaite, le Maréchal, les hommes de la Révolution nationale tenaient un langage dans lequel Emmanuel Mounier ne pouvait pas ne pas retrouver nombre de ses idées. D'où les controverses sur les sentiments et les prises de position de Mounier en 1940-1941. D'où la présence d'hommes aujourd'hui encore respectés, voire révérés, tel Hubert Beuve-Méry, dans des organisations subventionnées par Vichy, par exemple Uriage. Celui qui voudrait instruire le procès de la politique de Mounier n'a qu'à lire l'ouvrage rédigé par un fidèle d'Esprit, Michel Winock. Dunoyer de Segonzac, admirable combattant, demeura longtemps maréchaliste, en dépit des lois « infâmes ».

Je ne reproche pas à Bernard-Henri Lévy de mettre en lumière la parenté entre certains thèmes d'Esprit ou d'Ordre nouveau et ceux de la Révolution nationale. Il n'en résulte pas
qu'Emmanuel Mounier ait adhéré à l'ensemble du vichysme et célèbre le culte du Maréchal. L'argent d'Uriage venait de Vichy (celui du "Figaro » partiellement aussi), Beuve-Méry ne fléchit jamais dans son opposition radicale au national-socialisme. Chacun peut apprécier librement Uriage, « qui tient de l'histoire de la chevalerie, du roman d'éducation, du grand jeu secret de la fondation d'un ordre monastique, sur un fond d'idéalisme moral qui peut nous paraître un peu naïf et grandiloquent, et aussi passablement pétainiste, mais qui fut, notons-le, commun au vichysme et à la Résistance » (JeanMichel Jeanneney et Jacques Julliard).

Ce qui m'irrite, c'est le style dans lequel Bernard-Henri Lévy évoque ces moments tragiques de l'Histoire de France, sans la moindre compréhension des cas de conscience qui se posèrent à d'innombrables bons Français. Ceux qui passèrent, comme Mounier, de longs mois dans les prisons, accusés d'incarner un certain esprit de résistance, ceux d'Uriage, qui partirent tous ensemble dans le maquis les armes à la main, ont droit au moins à un certain respect. Il est légitime de critiquer les décisions qu'ils ont prises, les textes qu'ils ont écrits et publiés entre 1940 et 1942 ou 1945 : encore convient-il de les critiquer sérieusement, sans oublier les circonstances, sans excommunier le nationalisme de Péguy ou la pensée communautaire, quel que soit le jugement que l'on porte sur eux.

Nous savions depuis longtemps que la pensée raciste et l'antisémitisme ne sont pas monopole de l'Allemagne. Lequel des deux pays fut en avance sur l'autre ? Lequel influa le plus sur l'autre ? Sur toutes ces questions, des études sérieuses nous instruiraient, mais non pas l'utilisation, plus ou moins fantaisiste, des livres sérieux auxquels Bernard-Henri Lévy emprunte sa documentation.

Je m'en tiendrai à un point, à vrai dire essentiel. Qu'est-ce que cette idéologie française que ce livre s'efforce d'amener au jour en la baptisant? L'idéologie française, multiforme, insaisissable, partout présente, se situe aussi bien à droite qu'à gauche. Proudhon est farouchement antisémite. Maurras aussi, à l'autre extrémité de 
l'éventail. L'argent se confond avec le judaïsme, et la dénonciation de l'un et de l'autre se retrouve, presque identique, dans la littérature contre-révolutionnaire et dans la littérature socialiste. ("La Question juive », le texte le plus antisémite de Marx, illustre cette confusion volontaire.) Tout cela n'est pas neuf et nullement caractéristique de la France.

En quoi consiste la francité de cette idéologie, $\mathrm{du}$ « fascisme aux couleur de la France»? Quels sont les traits communs à Proudhon, Barrès, Maurras, Sorel, Péguy, Bernanos ? Je ne vois d'autre réponse que celle-ci : ils détestent la démocratie individualiste, liée au capitalisme, la République bourgeoise et libérale, celle de Benda (avant son ralliement au communisme) et de Bernard-Henri Lévy. Le patriotisme charnel, les communautés concrètes, les tendances à la vision organiciste du lien social, Bernard-Henri Lévy, lui, déteste ces manières de penser et de sentir ; il se fait inquisiteur et rejette dans la nuit, dans la "France noire », au hasard de ses lectures et de ses citations, Péguy et Bernanos, bien d'autres qui s'étonneraient de se retrouver en pareille compagnie. Simone Weil aurait mérité de figurer dans cette galerie des ancêtres.

Si l'on objectait à Bernard-Henri Lévy qu'il viole toutes les règles de l'interprétation honnête et de la méthode historique, il répondrait avec arrogance qu'il se moque des pions de l'Université. Mais peutêtre consentira-t-il à réfléchir un instant sur un fait indiscutable : le fascisme n'a jamais « pris » en France, comme une mayonnaise ne prend pas. Les idéologies des années 30, de type communautaire, anti-individualiste, n'ont jamais débouché en dehors des cénacles de l'intelligentsia parisienne. Elles ont accédé au pouvoir à la faveur d'une catastrophe nationale. Là encore elles sont demeurées un mixte de traditionalisme et de parafascisme.

Les " rénovateurs ", en quête d'une nouvelle droite, rompent avec l'héritage de la contrerévolution qui paralysa le développement d'un vrai fascisme. Les tentatives d'unir le nationalisme au socialisme ne manquèrent pas, mais il n'y eut pas de national-socialisme, et les Français, avant 1940 comme après 1945, votèrent pour la République et la démocratie parlementaire.
J'en viens à l'épilogue, raison d'être de cet article. Il se peut que certains adversaires de Bernard-Henri Lévy lui fassent payer son succès trop rapide, ses lecteurs trop nombreux. Je n'en veux pas à Bernard-Henri Lévy, et je garde la nostalgie du jeune homme que j'ai connu, il y a quelques années, pas encore guindé dans son personnage, tout au contraire disponible, comblé à la naissance par les dons des fées et les faveurs de la société. Je fus sensible à son talent, à son charme, et, je le crus, à la noblesse de ses sentiments, quand, à la suite d'un entretien avec moi, il rédigea l'interview publiée au «Nouvel Observateur » avec un tact sans faute.

Le voilà maintenant Fouquier-Tinville, lui qui prêche la démocratie. Il oublie que la démocratie devient aisément, elle aussi, inquisitoire, sinon totalitaire. Juif comme moi, il exclut de la France et rejette dans la France noire d'innombrables écrivains ou penseurs de notre commune patrie.

Nombre de Juifs, en France, se sentent de nouveau guettés par l'antisémitisme et, comme des êtres "choqués ", ils amplifient par leurs réactions le danger, plus ou moins illusoire, qu'ils affrontent. Que leur dit ce livre ? Que le péril est partout, que l'idéologie française les condamne à un combat de chaque instant contre un ennemi installé dans l'inconscient de millions de leurs concitoyens. Des Français non juifs en concluront que les Juifs sont encore plus différents des autres Français qu'ils ne l'imaginaient, puisqu'un auteur acclamé par les organisations juives se révèle incapable de comprendre tant d'expressions de la pensée française, au point de les mettre au ban de la France.

Il nous annonce la vérité pour que la nation française connaisse et surmonte son passé, il jette du sel sur toutes les plaies mal cicatrisées. Par son hystérie, il va nourrir l'hystérie d'une fraction de la communauté juive, déjà portée aux paroles et aux actes du délire. Un œuvre d'intérêt public, écrivait en conclusion le compte rendu du «Nouvel Observateur ». Intérêt public ou danger public? 